\title{
Direct and Inverse Spectral Problems for Rank-One Perturbations of Self-adjoint Operators
}

\author{
Oles Dobosevych@ and Rostyslav Hryniv@
}

\begin{abstract}
For a given self-adjoint operator $A$ with discrete spectrum, we completely characterise possible eigenvalues of its rank-one perturbations $B$ and discuss the inverse problem of reconstructing $B$ from its spectrum.
\end{abstract}

Mathematics Subject Classification. Primary: 47A55; Secondary: 47A10, 15A18, 15A60.

Keywords. Operators, Rank-one perturbations, Non-simple eigenvalues, Eigenvalue asymptotics.

\section{Introduction}

The main aim of this paper is to give a complete answer to the question, what spectra rank-one perturbations $B=A+\langle\cdot, \varphi\rangle \psi$ of a given self-adjoint operator $A$ with simple discrete spectrum may have. There are several reasons why this question is of interest. Firstly, such perturbations lead to the explicit formulae of perturbation theory and thus many related questions can be fully answered. Secondly, despite its simplicity, the model offers extremely rich family of perturbed spectra. Namely, the main results of this paper show that, apart from the prescribed asymptotic distribution of eigenvalues, the spectrum of a rank-one perturbation $B$ of $A$ might become arbitrary-in particular, it may get eigenvalues of arbitrarily prescribed multiplicities in an arbitrarily prescribed finite set of complex points. In addition, we suggest an explicit method of constructing rank-one perturbations of $A$ with a given admissible spectrum.

Similar questions in finite-dimensional case have been studied since 1990-ies. In particular, Krupnik proved in [23] that for any two sequences $\lambda_{1}, \lambda_{2}, \ldots, \lambda_{n}$ and $\mu_{1}, \mu_{2}, \ldots, \mu_{n}$ of complex numbers there exists an $n \times n$ matrix $A$ and its rank-one perturbation $B$ such that $\lambda_{j}$ are eigenvalues of $A$ and $\mu_{j}$ those of $B$, all counted with multiplicities; moreover, that statement 
was then further specified for the classes of Hermitian, unitary, and normal matrices. Savchenko [35] studied the changes in the Jordan structure of $A$ under a rank-one perturbation and found out that, generically, in each root subspace, only the longest Jordan chain splits. For low-rank perturbations, that result was further generalised in [36] and independently in [33]. In [17], the number of distinct eigenvalues of a matrix $B$ was estimated in terms of some spectral characteristics of $A$ and the rank of the perturbation. One should mention that earlier, Hörmander and Melin [21] explained similar effects of rank-one perturbations in an infinite-dimensional setting; recently, Behrndt a.o. [12] discussed possible changes to Jordan structure of an arbitrary linear operator $A$ in a Banach space under general finite-rank perturbations.

For structured matrices and matrix pencils, a detailed rank-one perturbation theory and its application in the control theory was recently developed in a series of papers by Mehl a.o. [27-32,38]. The results established therein include e.g. changes in the Jordan structure of $A$ under perturbation within classes of matrices enjoying certain real or complex Hamiltonian symmetry $[27,29,31]$, or for $H$-Hermitian matrices, with (skew-)Hermitian $H$, using the canonical form of the pair $(B, H)[28,30]$. Rank-one perturbations of matrix pencils and an important eigenvalue placement problem were studied e.g. in $[11,19,32]$, while a more general perturbation theory for structured matrices was outlined in the recent paper [38].

The cited results are mostly essentially finite-dimensional in the sense that their methods do not allow straightforward generalization to the infinitedimensional case (see, however, $[12,21]$ ). The latter has been studied within the general spectral theory for bounded or unbounded operators in infinitedimensional Banach or Hilbert spaces [22]. For instance, a comprehensive spectral analysis of rank-one perturbations of unbounded self-adjoint operators is carried out in [37], where a detailed characterization of discrete, absolutely continuous, and singularly continuous spectra of the perturbation $B$ is given. A thorough overview of the theory of Schrödinger operators under singular point perturbations (formally corresponding to additive Dirac deltafunctions and their derivatives) is given in the monographs by Albeverio a.o. $[2,9]$, suggesting also comprehensive reference lists. Much attention has been paid to the so-called singular and super-singular rank-one or finite-rank perturbations of self-adjoint operators, where the functions $\varphi$ and $\psi$ belong to the scales of Hilbert spaces $\operatorname{dom}\left(A^{\alpha}\right)$ with negative $\alpha$, see e.g. $[3-8,10,16,18,24-$ $26]$; in this case, a typical approach is through the Krein extension theory of self-adjoint operators. Rank-one and finite-rank perturbations of self-adjoint operators in Krein spaces have been recently discussed in e.g. [13,14].

Despite the extensive research in the area, there seems to be no complete understanding what spectra rank-one perturbations of a given operator $A$ can produce. As the earlier research demonstrates (cf. $[2,9,37]$ ), the question is quite non-trivial even for self-adjoint perturbations of a self-adjoint operator $A$, and thus necessarily much more complicated for generic rank-one perturbations. In our previous work [15], we described local spectral properties of rank-one perturbations of a self-adjoint operator with discrete spectrum and proved that the eigenvalues of $B$ are asymptotically close to those 
of $A$ at infinity. In particular, it was shown therein that such a perturbation can possess eigenvalues of arbitrarily prescribed multiplicities at any finite set of complex numbers thus significantly extending the finite-dimensional result of [23]. Recently, similar properties were derived in [20] for the class of $\mathcal{P} \mathcal{T}$-symmetric perturbations.

The main aim of the present paper is to suggest a complete characterization of possible spectra of rank-one perturbations of a given self-adjoint operator $A$ with simple discrete spectrum. More exactly, with $\lambda_{n}$ denoting the eigenvalues of $A$, Theorem 3.1 states that the eigenvalues of a rank-one perturbation $B$ can be labelled as $\mu_{n}$ (counting with multiplicities) so that the sum of all offsets $\left|\mu_{n}-\lambda_{n}\right|$ is finite. Moreover, Theorem 4.1 proves that every sequence $\mu_{n}$ with this property can be a spectrum for such a $B$ and, in addition, suggests a method for constructing all such rank-one perturbations $B$; uniqueness of $B$ is discussed in Corollary 5.4.

The structure of the paper is as follows. In the next section, we collect some basic spectral properties of the rank-one perturbations $B$ and review the results of [15]. In Sect. 3, the asymptotic distribution of eigenvalues of $B$ is studied and, in particular, summability of the offsets $\left|\mu_{n}-\lambda_{n}\right|$ is proved. Sufficiency of this condition, as well as an algorithm for constructing a rankone perturbation $B$ with a prescribed admissible spectrum are established in Sect. 4. Finally, in Sect. 5, we give two examples, comment on uniqueness of $B$ when $\phi$ or $\psi$ is fixed, and discuss straightforward generalisations of the main results to wider classes of the operators $A$.

\section{Preliminaries}

In this section, we collect some properties of the rank-one perturbations of self-adjoint operators $A$ acting in a fixed separable (infinite-dimensional) Hilbert space $H$ established in [15] that will be used to prove the main results of this work.

Throughout the paper, we shall assume that

(A1) the operator $A$ is self-adjoint and has simple discrete spectrum.

The operator $A$ is necessarily unbounded but it may be bounded below or above; without loss of generality, in this case we assume that $A$ is bounded below (otherwise, we just replace $A$ with $-A$ ). Under these assumptions, the spectrum of $A$ consists of simple real eigenvalues that can be listed in increasing order as $\lambda_{n}, n \in I$, with $I=\mathbb{N}$ if $A$ is bounded below and $I=\mathbb{Z}$ otherwise. Keeping in mind the most important and interesting applications to the differential operators, we make an additional assumption that

(A2) the eigenvalues of $A$ are $d$-separated, i.e.,

$$
\inf _{n \in I}\left|\lambda_{n+1}-\lambda_{n}\right|=: d>0 .
$$

Next, the operator $B$ is a rank-one perturbation of the operator $A$, i.e.,

$$
B=A+\langle\cdot, \varphi\rangle \psi
$$


with fixed non-zero vectors $\varphi$ and $\psi$ in $H$ and with $\langle\cdot, \cdot\rangle$ denoting the scalar product in $H$. Clearly, $B$ is well defined and closed on its natural domain $\operatorname{dom}(B)$ equal to $\operatorname{dom}(A)$. Further, for $\lambda \in \rho(A)$, we introduce the characteristic function

$$
F(\lambda):=\left\langle(A-\lambda)^{-1} \psi, \varphi\right\rangle+1 .
$$

This function appears in the Krein resolvent formula for $B[9,15]$, and its zeros characterise the spectrum of $B$.

To be more specific, we denote by $v_{n}$ a normalised eigenvector of $A$ corresponding to the eigenvalue $\lambda_{n}$; then the set $\left\{v_{n}\right\}_{n \in I}$ forms an orthonormal basis of $H$, and we let $a_{n}$ and $b_{n}$ be the corresponding Fourier coefficients of the vectors $\varphi$ and $\psi$, so that

$$
\varphi=\sum_{k \in I} a_{k} v_{k}, \quad \psi=\sum_{k \in I} b_{k} v_{k} .
$$

Now we set

$$
I_{0}:=\left\{n \in I \mid a_{n} b_{n}=0\right\}, \quad I_{1}:=\left\{n \in I \mid a_{n} b_{n} \neq 0\right\}
$$

and $\sigma_{j}(A):=\left\{\lambda_{n} \mid n \in I_{j}\right\}$; then $\sigma_{0}(A)=\sigma_{0}(B):=\sigma(A) \cap \sigma(B)$ is the common part of the spectra of $A$ and $B$, while the spectrum of $B$ in $\mathbb{C} \backslash \sigma_{0}(A)$ coincides with the set of zeros of $F$.

In fact, the function $F$ also characterises eigenvalue multiplicities of the operator $B$. We recall that the geometric multiplicity of an eigenvalue $\lambda$ of $B$ is the dimension of the null-space of the operator $B-\lambda$, while its algebraic multiplicity is the dimension of the corresponding root subspace, i.e., of the set of all $y \in \operatorname{dom}(B)$ such that $(B-\lambda)^{k} y=0$ for some $k \in \mathbb{N}$. Next, by the spectral theorem for $A$, the characteristic function $F$ of (2.3) can be written as ${ }^{1}$

$$
F(z)=\sum_{k \in I_{1}} \frac{\overline{a_{k}} b_{k}}{\lambda_{k}-z}+1
$$

and thus can be analytically extended to $\sigma_{0}(A)$; we keep the notation $F$ for this extension.

As proved in [15], the geometric multiplicity of every eigenvalue $\mu$ of $B$ is at most 2 ; multiplicity 2 is only possible when $\mu \in \sigma_{0}(A)$ (i.e., when $\mu=\lambda_{n}$ for some $\left.n \in I_{0}\right)$ and, in addition, $a_{n}=b_{n}=F\left(\lambda_{n}\right)=0$. We also observe that when $a_{n}=b_{n}=0$, then the subspace $\operatorname{ls}\left\{v_{n}\right\}$ is invariant under both $B$ and $B^{*}$ and thus is reducing for $B$. Denoting by $H_{0}$ the closed linear span of all such subspaces, we conclude that $H_{0}$ and $H \ominus H_{0}$ are reducing for $B$ and the operators $A$ and $B$ coincide on $H_{0}$. For that reason, only the part of $B$ in $H \ominus H_{0}$ is of interest, and, without loss of generality, we may assume that $H_{0}=\{0\}$.

Under such an assumption, every eigenvalue $\mu$ of $B$ is geometrically simple and the main results of [15] can be summarised as follows:

\footnotetext{
${ }^{1}$ In what follows, the summations and products over the index sets that are not bounded from below or above are understood in the principal value sense
} 
(a) the algebraic multiplicity $m$ of an eigenvalue $\mu \in \sigma(B) \backslash \sigma_{0}(B)$ coincides with the multiplicity $l$ of $z=\mu$ as a zero of $F$;

(b) if $\mu \in \sigma_{0}(B)$, then the above multiplicities $m$ and $l$ satisfy the relation $m=l+1$;

(c) for any $n$-tuple $z_{1}, z_{2}, \ldots, z_{n}$ of pairwise distinct complex numbers and any $n$-tuple $m_{1}, m_{2}, \ldots, m_{n}$ of natural numbers, there exists a rank-one perturbation $B$ of $A$ such that every $z_{j}$ is an eigenvalue of $B$ of algebraic multiplicity $m_{j}$;

(d) the eigenvalues of $B$ can be enumerated as $\mu_{n}, n \in I$, in such a way that $\mu_{n}-\lambda_{n} \rightarrow 0$ as $|n| \rightarrow \infty$; in particular, $B$ has at most finitely many non-simple eigenvalues.

Property (c) implies that locally the spectrum of $B$ can be arbitrary while (d) describes the asymptotic behaviour of the eigenvalues of $B$ at infinity. The main purpose of this research is to give a complete characterisation of the possible spectra of $A$ under rank-one perturbations by refining the asymptotics of $\mu_{n}$, cf. Theorems 3.1 and 4.1. By virtue of (a) and (b), this task amounts to the study of zero distribution of the characteristic function $F$ of (2.3), which will be carried out in Sects. 3 and 4 .

\section{Eigenvalue Distribution of the Operator $B$}

In this section, we shall discuss eigenvalue distribution of the rank-one perturbation $B$ of $A$ given by (2.2). As explained in the previous section, the spectrum of $B$ consists of two parts: $\sigma_{0}(B)=\sigma(A) \cap \sigma(B)$, which is the common part of the spectra of $A$ and $B$, and $\sigma_{1}(B):=\sigma(B) \backslash \sigma_{0}(B)$, which is the set of zeros of the characteristic function

$$
F(z)=\sum_{n \in I_{1}} \frac{\overline{a_{n}} b_{n}}{\lambda_{n}-z}+1
$$

in the domain $\mathbb{C} \backslash \sigma_{1}(A)$; moreover, the algebraic multiplicity of an eigenvalue $\mu \in \sigma(B)$ is determined by its multiplicity as a zero of the characteristic function $F$.

The main result of this section is given by the following theorem.

Theorem 3.1. The eigenvalues of the operator $B$ can be labelled as $\mu_{n}, n \in I$, in such a way that the series

$$
\sum_{n \in I}\left|\mu_{n}-\lambda_{n}\right|
$$

converges. In particular, all but finitely many eigenvalues of $B$ are simple.

First we shall show that large enough elements of $\sigma_{1}(B)$ are located near $\sigma_{1}(A)$, which will enable their proper enumeration. To begin with, for $k \in I_{1}$ we define the functions $G_{k}$ and $H_{k}$ by the formulas ${ }^{2}$

$$
G_{k}(z)=\frac{\overline{a_{k}} b_{k}}{\lambda_{k}-z}+1, \quad H_{k}(z)=\sum_{|n| \leq k}^{(1)} \frac{\overline{a_{n}} b_{n}}{\lambda_{n}-z}+1
$$

\footnotetext{
${ }^{2}$ Throughout the paper, the symbol $\sum^{(1)}$ will denote summation over the index set $I_{1}$
} 
and introduce the sets

$$
\begin{aligned}
& Q_{k}:=\left\{z \in \mathbb{C} \mid \operatorname{Re}(z), \operatorname{Im}(z) \in\left(\lambda_{-|k|}-\frac{d}{2}, \lambda_{|k|}+\frac{d}{2}\right)\right\}, \\
& R_{k}:=\left\{z \in \mathbb{C}|| z-\lambda_{k} \mid<\frac{d}{2}\right\},
\end{aligned}
$$

where we replace $\lambda_{-|k|}$ with $-\lambda_{|k|}$ if $I=\mathbb{N}$. Due to the assumption (A2) the sets $R_{k}$ are pairwise disjoint and also $R_{k} \cap Q_{n}=\emptyset$ if $|k|>|n|$.

Lemma 3.2. For every $\varepsilon>0$ there exist integers $K_{\varepsilon}>0$ and $K_{\varepsilon}^{\prime}>K_{\varepsilon}$ such that the following holds:

(a) for every $k$ with $|k|>K_{\varepsilon}$ and every $z \in \overline{R_{k}}=\partial R_{k} \cup R_{k}$

$$
\sum_{\substack{|n|>K_{\varepsilon} \\ n \neq k}}^{(1)}\left|\frac{\overline{a_{n}} b_{n}}{\lambda_{n}-z}\right|<\frac{2 \varepsilon}{d}
$$

(b) for every $z \in \mathbb{C} \backslash Q_{K_{\varepsilon}^{\prime}}$

$$
\sum_{|n| \leq K_{\varepsilon}}^{(1)}\left|\frac{\overline{a_{n}} b_{n}}{\lambda_{n}-z}\right|<\varepsilon
$$

Proof. The sequences $\left(a_{n}\right)_{n \in I}$ and $\left(b_{n}\right)_{n \in I}$ of the Fourier coefficients of the vectors $\varphi$ and $\psi$ are square summable, so that, by the Cauchy-BunyakowskySchwarz inequality,

$$
\sum_{n \in I_{1}}\left|\overline{a_{n}} b_{n}\right|<\infty
$$

Therefore, for every $\varepsilon>0$ there exists a $K_{\varepsilon}$ such that

$$
\sum_{|n|>K_{\varepsilon}}^{(1)}\left|\overline{a_{n}} b_{n}\right|<\varepsilon
$$

Take a $k$ satisfying $|k|>K_{\varepsilon}$; then by virtue of Assumption (A2) for every $z \in \overline{R_{k}}$ and every $n \neq k$ we get $\left|\lambda_{n}-z\right| \geq \frac{d}{2}$, and therefore (3.3) holds.

For part (b), note that $\left|\lambda_{n}-z\right|>\left(K_{\varepsilon}^{\prime}-K_{\varepsilon}\right) d$ if $|n| \leq K_{\varepsilon}$ and $z \in \mathbb{C} \backslash Q_{k}$ with $|k| \geq K_{\varepsilon}^{\prime}>K_{\varepsilon}$; therefore, by choosing $K_{\varepsilon}^{\prime}$ large enough, we arrive at (3.4).

Corollary 3.3. Take $\varepsilon:=d /(2+d)$ and $K_{\varepsilon}^{\prime}$ as in the above lemma; then

$$
\sigma(B) \subset Q_{K_{\varepsilon}^{\prime}} \cup\left(\bigcup_{n \in I} R_{n}\right) \text {. }
$$

Indeed, it suffices to note that if $z$ is outside $Q_{K_{\varepsilon}^{\prime}}$ and every $R_{n}, n \in I$, then $\left|\lambda_{n}-z\right| \geq d / 2$, so that

$$
\sum_{|n|>K_{\varepsilon}}^{(1)}\left|\frac{\overline{a_{n}} b_{n}}{\lambda_{n}-z}\right|<\frac{2 \varepsilon}{d}
$$

which together with part (b) of that lemma shows that

$$
|F(z)| \geq 1-\sum_{|n| \leq K_{\varepsilon}}^{(1)}\left|\frac{\overline{a_{n}} b_{n}}{\lambda_{n}-z}\right|-\sum_{|n|>K_{\varepsilon}}^{(1)}\left|\frac{\overline{a_{n}} b_{n}}{\lambda_{n}-z}\right|>1-\varepsilon(1+2 / d)=0 .
$$


Lemma 3.4. There exists a $K>0$ such that for all $k \in I_{1}$ with $|k|>K$ the following holds:

(a) the function $F$ has exactly one zero in $R_{k}$;

(b) the functions $H_{k}$ and $F$ have the same number of zeros in $Q_{k}$.

Proof. Fix an $\varepsilon \in(0, d / 2)$ such that

$$
\varepsilon\left(1+\frac{4}{d}\right)<1
$$

we shall show that (a) and (b) hold for $K=K_{\varepsilon}^{\prime}$ of Lemma 3.2.

If $k$ satisfies $|k|>K$, then by Lemma 3.2 for every $z \in \partial R_{k}$ we get

$$
\left|F(z)-G_{k}(z)\right| \leq \sum_{|n| \leq K_{\varepsilon}}^{(1)}\left|\frac{\overline{a_{n}} b_{n}}{\lambda_{n}-z}\right|+\sum_{\substack{|n|>K_{\varepsilon} \\ n \neq k}}^{(1)}\left|\frac{\overline{a_{n}} b_{n}}{\lambda_{n}-z}\right|<\varepsilon+\frac{2 \varepsilon}{d} .
$$

On the other hand, $\left|\overline{a_{k}} b_{k}\right|<\varepsilon$ if $k \in I_{1}$ satisfies $|k|>K>K_{\varepsilon}$, and then

$$
\left|G_{k}(z)\right| \geq 1-\left|\frac{\overline{a_{k}} b_{k}}{z-\lambda_{k}}\right|>1-\frac{2 \varepsilon}{d}
$$

for all $z \in \partial R_{k}$. By the choice of $\varepsilon$ we conclude that then

$$
\left|G_{k}(z)\right|>\left|F(z)-G_{k}(z)\right|
$$

for all such $z$. As the functions $G_{k}$ and $F$ both have the same number of poles in $R_{k}$ (namely, a simple pole at $\lambda_{k}$ ), by estimate (3.5) and Rouché's theorem they have the same number of zeros in the set $R_{k}$. By virtue of the inequality $\left|\overline{a_{k}} b_{k}\right|<\varepsilon<d / 2$, the unique zero $z=\lambda_{k}+\overline{a_{k}} b_{k}$ of the function $G_{k}$ belongs to the circle $R_{k}$ for all $k \in I_{1}$ with $|k|>K$, and thus the function $F$ has exactly one zero in $R_{k}$ for such $k$ as well. This completes the proof of part (a).

Next, by the definition of the set $Q_{k}$, it holds that $\left|\lambda_{n}-z\right| \geq \frac{d}{2}$ if $z \in \partial Q_{k}$ and $|n|>|k|$. By the choice of the number $K_{\varepsilon}$, we find that

$$
\left|F(z)-H_{k}(z)\right| \leq \sum_{|n|>|k|}^{(1)}\left|\frac{\overline{a_{n}} b_{n}}{\lambda_{n}-z}\right|<\frac{2 \varepsilon}{d}
$$

and

$$
\sum_{K_{\varepsilon}<|n| \leq|k|}^{(1)}\left|\frac{\overline{a_{n}} b_{n}}{\lambda_{n}-z}\right|<\frac{2 \varepsilon}{d}
$$

if $|k|>K_{\varepsilon}$ and $z \in \partial Q_{k}$. Also, by part (b) of Lemma 3.2 we have

$$
\sum_{|n| \leq K_{\varepsilon}}^{(1)}\left|\frac{\overline{a_{n}} b_{n}}{\lambda_{n}-z}\right|<\varepsilon
$$

as soon as $|k|>K$ and $z \in \partial Q_{k}$. Combining estimates (3.6) and (3.7), we conclude that

$$
\left|H_{k}(z)\right| \geq 1-\sum_{|n| \leq k}^{(1)}\left|\frac{\overline{a_{n}} b_{n}}{\lambda_{n}-z}\right|>1-\varepsilon-\frac{2 \varepsilon}{d}
$$

for all $k$ with $|k|>K$ and all $z \in \partial Q_{k}$. 
It follows that for $k$ with $|k|>K$ and for all $z \in \partial Q_{k}$

$$
\left|H_{k}(z)\right|>\left|F(z)-H_{k}(z)\right| \text {. }
$$

Since the functions $H_{k}$ and $F$ have the same poles in $Q_{k}$ (namely, simple poles $\lambda_{n}$ for $n \in I_{1}$ with $|n| \leq|k|$ ), we conclude by Rouché's theorem that they have the same number of zeros in $Q_{k}$ if $|k|>K$.

Remark 3.5. Take $k$ larger than $K$ of the above lemma and denote by $N_{k}$ the cardinality of the set $\sigma_{1}(A) \cap Q_{k}$. The function $H_{k}$ is a ratio of two polynomials of degree $N_{k}$ and due to (3.8) all its zeros are in $Q_{k}$. Therefore, the function $F$ has precisely $N_{k}$ zeros in $Q_{k}$ counting with multiplicities.

Corollary 3.6. The zeros of $F$ in $\mathbb{C} \backslash \sigma_{0}(A)$ can be labelled (counting with multiplicities) as $\mu_{k}$ with $k \in I_{1}$ in such a way that $\left|\mu_{k}-\lambda_{k}\right|<\frac{d}{2}$ for all $k \in I_{1}$ with $|k|>K$.

Recalling the results of the previous section on relation between the eigenvalues of $B$ and zeros of the function $F$ in $\mathbb{C} \backslash \sigma_{1}(A)$, we arrive at the following conclusion.

Corollary 3.7. Eigenvalues of the operator $B$ can be labelled (counting with multiplicities) as $\mu_{k}$ with $k \in I$ in such a way that $\left|\mu_{k}-\lambda_{k}\right|<\frac{d}{2}$ when $|k|>K, K$ being the constant of Lemma 3.4 .

Combining the above corollary with Lemma 4.3 of [15], we conclude that $\left|\mu_{k}-\lambda_{k}\right| \rightarrow 0$ as $|k|$ goes to infinity, cf. Theorem 4.7(ii) of [15]. However, the estimates established above will enable us to prove a stronger statement of Theorem 3.1 on the asymptotics of $\left|\mu_{k}-\lambda_{k}\right|$.

Proof of Theorem 3.1. We fix an enumeration of $\mu_{k}$ as in Corollary 3.7. Then $\mu_{k}=\lambda_{k}$ for all $k \in I_{0}$ with large enough $|k|$, whence it suffices to prove that the series

$$
\sum_{n \in I_{1}}\left|\mu_{n}-\lambda_{n}\right|
$$

is convergent.

We take $\varepsilon$ and $K$ as in Lemma 3.4; then, according to Corollary 3.7, for every $k \in I_{1}$ with $|k|>K$ the eigenvalue $\mu_{k} \in R_{k}$ is a zero of $F$, so that

$$
F\left(\mu_{k}\right)=\sum_{|n| \leq K_{\varepsilon}}^{(1)} \frac{\overline{a_{n}} b_{n}}{\mu_{k}-\lambda_{n}}+\sum_{\substack{|n|>K_{\varepsilon} \\ n \neq k}}^{(1)} \frac{\overline{a_{n}} b_{n}}{\mu_{k}-\lambda_{n}}+\frac{\overline{a_{k}} b_{k}}{\mu_{k}-\lambda_{k}}+1=0
$$

and

$$
\left|\frac{\overline{a_{k}} b_{k}}{\mu_{k}-\lambda_{k}}\right|>1-\sum_{|n| \leq K_{\varepsilon}}^{(1)}\left|\frac{\overline{a_{n}} b_{n}}{\lambda_{n}-\mu_{k}}\right|-\sum_{\substack{|n|>K_{\varepsilon} \\ n \neq k}}^{(1)}\left|\frac{\overline{a_{n}} b_{n}}{\lambda_{n}-\mu_{k}}\right| .
$$

By virtue of Lemma 3.4 we conclude that

$$
\left|\frac{\overline{a_{k}} b_{k}}{\mu_{k}-\lambda_{k}}\right|>1-\varepsilon-\frac{2 \varepsilon}{d}
$$


as $1-\varepsilon-\frac{2 \varepsilon}{d}>\frac{2 \varepsilon}{d}$, we find that

$$
\left|\mu_{k}-\lambda_{k}\right|<\frac{d}{2 \varepsilon}\left|\overline{a_{k}} b_{k}\right|
$$

for all $k \in I_{1}$ with $|k|>K$. Since the series $\sum_{n \in I_{1}}\left|\bar{a}_{n} b_{n}\right|$ is convergent, the same is true of the series $\sum_{n \in I_{1}}\left|\mu_{n}-\lambda_{n}\right|$.

\section{Inverse Spectral Problem}

The purpose of this section is to study the inverse spectral problem, namely, the problem of reconstructing the operator $B$ from its spectrum $\left(\mu_{n}\right)_{n \in I}$ assuming that the operator $A$ is known.

More generally, let the operator $A$ satisfy assumptions (A1) and (A2), i.e., is self-adjoint and has a simple discrete spectrum $\left(\lambda_{n}\right)_{n \in I}$ that is $d$ separated as in (2.1). Our aim is to find necessary and sufficient conditions that another sequence $\left(\nu_{n}\right)_{n \in I}$ of complex numbers must satisfy so that it could be a spectrum (counting with multiplicities) of an operator $B$ of the form (2.2). Also, we want to suggest an algorithm of constructing the operator $B$ (i.e., the function $\varphi$ and $\psi$ ) and investigate the uniqueness of such $\varphi$ and $\psi$.

The latter question can be answered straight ahead. Indeed, if the inverse problem for a sequence $\left(\nu_{n}\right)_{n \in I}$ has a solution, then it has many solutions. In fact, if

$$
B_{j}=A+\left\langle\cdot, \varphi_{j}\right\rangle \psi_{j}, \quad j=1,2,
$$

and vectors $\varphi_{1}, \varphi_{2}, \psi_{1}, \psi_{2}$ satisfy

$$
\overline{\left\langle\varphi_{1}, v_{n}\right\rangle}\left\langle\psi_{1}, v_{n}\right\rangle=\overline{\left\langle\varphi_{2}, v_{n}\right\rangle}\left\langle\psi_{2}, v_{n}\right\rangle, \quad n \in I,
$$

then the spectra of $B_{1}$ and $B_{2}$ coincide counting with multiplicities. Therefore, in the inverse problem one can only restore the products $\bar{a}_{n} b_{n}$ of the Fourier coefficients of the functions $\varphi$ and $\psi$, which are the residues of the function $-F$ of (2.5).

The main result of this section is given by the following theorem.

Theorem 4.1. Assume that a sequence $\boldsymbol{\nu}$ of complex numbers can be enumerated as $\nu_{n}, n \in I$, in such a way that the series

$$
\sum_{n \in I}\left|\nu_{n}-\lambda_{n}\right|
$$

converges. Then there exist vectors $\varphi, \psi \in H$ such that the spectrum of $B$ coincides with $\boldsymbol{\nu}$ counting with multiplicities.

Let us denote by $I_{0}$ the set of indices $n \in I$ for which $\lambda_{n}$ appears in $\boldsymbol{\nu}$ and set $\Lambda_{0}:=\left\{\lambda_{n} \mid n \in I_{0}\right\}$. Convergence of the series (4.1) implies that for every $\varepsilon \in(0, d / 2)$ there exists a $K>0$ such that $\left|\nu_{n}-\lambda_{n}\right|<\varepsilon$ for all $n \in I$ with $|n|>K$. Therefore, if $n \in I_{0}$ and $|n|>K$, then $\nu_{n}=\lambda_{n}$, and without loss of generality we may assume that $\nu_{n}=\lambda_{n}$ for all $n \in I_{0}$. 
We also set $I_{1}:=I \backslash I_{0}, \Lambda_{1}:=\left\{\lambda_{n} \mid n \in I_{1}\right\}$, and introduce the function

$$
\tilde{F}(z):=\prod_{n \in I_{1}} \frac{\nu_{n}-z}{\lambda_{n}-z} .
$$

To show that $\tilde{F}$ is well defined, we take an arbitrary $\varepsilon \in(0, d / 2)$ and set

$$
R_{n}(\varepsilon):=\left\{z \in \mathbb{C}|| z-\lambda_{n} \mid<\varepsilon\right\}, \quad R(\varepsilon):=\mathbb{C} \backslash\left(\cup_{n \in I_{1}} R_{n}(\varepsilon)\right) .
$$

Then we have the following

Lemma 4.2. For each $\varepsilon \in(0, d / 2)$, the product in (4.2) converges uniformly in $R(\varepsilon)$.

Proof. It is enough to show that the series

$$
\sum_{n \in I_{1}} \log \left(1+\left|\frac{\nu_{n}-\lambda_{n}}{\lambda_{n}-z}\right|\right)
$$

converges uniformly on the set $R(\varepsilon)$. However, for $z \in R(\varepsilon)$ and $n \in I_{1}$ we get the estimate

$$
\log \left(1+\left|\frac{\nu_{n}-\lambda_{n}}{\lambda_{n}-z}\right|\right) \leq\left|\frac{\nu_{n}-\lambda_{n}}{\lambda_{n}-z}\right| \leq \frac{\left|\nu_{n}-\lambda_{n}\right|}{\varepsilon},
$$

which in view of the convergence of the series (4.1) and the Weierstrass M-test finishes the proof.

The Weierstrass $M$-test used in the above proof also justifies passage to the limit

$$
\lim _{u \rightarrow+\infty} \sum_{n \in I_{1}}\left|\frac{\nu_{n}-\lambda_{n}}{\lambda_{n}-i u}\right|=0
$$

as a result, we get

Corollary 4.3. There exists the limit

$$
\lim _{u \rightarrow+\infty} \tilde{F}(i u)=1 .
$$

The function $\tilde{F}$ is meromorphic in $\mathbb{C}$, and its residue at the point $\lambda_{n} \in \Lambda_{1}$ is

$$
-c_{n}=\lim _{z \rightarrow \lambda_{n}}\left(z-\lambda_{n}\right) \tilde{F}(z)=\left(\lambda_{n}-\nu_{n}\right) \prod_{\substack{m \in I_{1} \\ m \neq n}} \frac{\nu_{m}-\lambda_{n}}{\lambda_{m}-\lambda_{n}}
$$

Lemma 4.4. The series

$$
\sum_{n \in I_{1}}\left|c_{n}\right|
$$

converges. 
Proof. In view of (4.4), convergence of series (4.5) follows from convergence of the series

$$
\sum_{n \in I_{1}}\left|\lambda_{n}-\nu_{n}\right| \prod_{\substack{m \in I_{1} \\ m \neq n}}\left|\frac{\nu_{m}-\lambda_{n}}{\lambda_{m}-\lambda_{n}}\right|,
$$

and to establish the latter it is enough to show that the sequence

$$
\prod_{\substack{m \in I_{1} \\ m \neq n}}\left|\frac{\nu_{m}-\lambda_{n}}{\lambda_{m}-\lambda_{n}}\right|
$$

is bounded in $n \in I_{1}$.

Applying the same reasoning as in the proof of Lemma 4.2, we conclude that the sum of the series

$$
\begin{aligned}
\sum_{m \neq n}^{(1)} \log \left|\frac{\nu_{m}-\lambda_{n}}{\lambda_{m}-\lambda_{n}}\right| & \leq \sum_{m \neq n}^{(1)} \log \left(1+\left|\frac{\nu_{m}-\lambda_{m}}{\lambda_{m}-\lambda_{n}}\right|\right) \\
& \leq \sum_{m \neq n}^{(1)}\left|\frac{\nu_{m}-\lambda_{m}}{\lambda_{m}-\lambda_{n}}\right| \leq \frac{1}{d} \sum_{m \in I_{1}}\left|\nu_{m}-\lambda_{m}\right|
\end{aligned}
$$

has an $n$-independent bound, which implies that the sequence (4.6) is uniformly bounded.

In view of the above lemma, the series

$$
\sum_{n \in I_{1}} \frac{c_{n}}{\lambda_{n}-z}
$$

converges uniformly in $R(\varepsilon)$ for every $\varepsilon \in(0, d / 2)$. It follows that the function

$$
F(z):=1+\sum_{n \in I_{1}} \frac{c_{n}}{\lambda_{n}-z}
$$

is well defined and analytic in the set $\mathbb{C} \backslash \Lambda_{1}$ and has simple poles at the points $z \in \Lambda_{1}$. The Lebesgue dominated convergence theorem also implies that

$$
\lim _{u \rightarrow+\infty} F(i u)=1
$$

Lemma 4.5. The function $F-\tilde{F}$ is equal to zero identically in $\mathbb{C}$.

Proof. We set $G:=F-\tilde{F}$; then the function $G$ is meromorphic in $\mathbb{C}$ with possible single poles at the points $\Lambda_{1}$. However, as the residua of $F$ and $\tilde{F}$ at each point $z \in \Lambda_{1}$ coincide by construction, we conclude that the function $G$ has removable singularities at the points $z \in \Lambda_{1}$ and thus is entire. We next show that $G$ is uniformly bounded over $\mathbb{C}$ and thus is constant by the Liouville theorem; as

$$
\lim _{u \rightarrow+\infty} G(i u)=\lim _{u \rightarrow+\infty} F(i u)-\lim _{u \rightarrow+\infty} \tilde{F}(i u)=0
$$

this constant is zero, and thus the proof will be complete. 
For $k$ with large enough $|k|$, we take the square $Q_{k}$ of (3.2) and observe that for every $n \in I_{1}$ and $z \in \partial Q_{k}$ we have $\left|z-\lambda_{n}\right| \geq d / 2$; as a result, we conclude that

$$
\sup _{z \in \partial Q_{k}}|F(z)| \leq 1+\frac{2}{d} \sum_{n \in I_{1}}\left|c_{n}\right|:=C .
$$

Next, we note that for $\varepsilon \in(0, d / 2)$ the boundary $\partial Q_{k}$ of $Q_{k}$ lies in the set $R(\varepsilon)$. As in the proof of Lemma 4.2, we can derive the bound (cf. (4.3))

$$
\sup _{z \in \partial Q_{k}}|\tilde{F}(z)| \leq \exp \left\{\frac{1}{\varepsilon} \sum_{n \in I_{1}}\left|\nu_{n}-\lambda_{n}\right|\right\}:=\tilde{C} .
$$

Since the function $G$ is entire, it follows from the maximum modulus principle that

$$
|G(z)| \leq C+\tilde{C}
$$

inside every set $Q_{k}$ and thus for all $z \in \mathbb{C}$. Therefore, the function $G$ is bounded; as explained at the beginning of the proof, this implies the required result.

Proof of Theorem 4.1. Given any sequence $\boldsymbol{\nu}$ of complex numbers satisfying the assumption of the theorem, we construct the meromorphic function $\tilde{F}$ via (4.2). Next, we calculate the residua $-c_{n}$ of $\tilde{F}$ at the points $\lambda_{n} \in \Lambda_{1}$ via (4.4) and define sequences $\left(a_{n}\right)_{n \in I}$ and $\left(b_{n}\right)_{n \in I}$ via

$$
a_{n}:=\sqrt{\left|c_{n}\right|}, \quad b_{n}:=\sqrt{\left|c_{n}\right|} e^{i \arg c_{n}}, \quad n \in I_{1},
$$

and

$$
a_{n}:=1 /(1+|n|), \quad b_{n}=0, \quad n \in I_{0} .
$$

Since the sequence $\left(c_{n}\right)_{n \in I_{1}}$ is summable by Lemma 4.4 , it follows that the sequences $\left(a_{n}\right)_{n \in I}$ and $\left(b_{n}\right)_{n \in I}$ belong to $\ell_{2}(I)$. Therefore, there exist functions $\varphi$ and $\psi$ in the Hilbert space $\mathcal{H}$ whose Fourier coefficients in the basis $\left(v_{n}\right)_{n \in I}$ are equal to $a_{n}$ and $b_{n}$, respectively.

We now consider the operator $B$ of the form (2.2) with the functions $\varphi$ and $\psi$ just introduced and conclude by virtue of Lemma 4.5 that the corresponding characteristic function $F$ of $(2.5)$ coincides with $\tilde{F}$. Therefore, zeros of $F$ are precisely the elements of the subsequence $\boldsymbol{\nu}_{1}:=\left(\nu_{n}\right)_{n \in I_{1}}$, both counting multiplicity; namely, if a number $\nu$ occurs $k$ times in $\boldsymbol{\nu}_{1}$, it is a zero of $F$ of multiplicity $k$. The analysis of the paper [15] summarised in Sect. 2 shows that each element $\nu$ of $\nu$ is an eigenvalue of $B$ and its multiplicity is equal to the number of times $\nu$ is repeated in the sequence $\nu$.

The above proof also suggests an algorithm of constructing a particular operator $B$ whose spectrum corresponds to a sequence $\boldsymbol{\nu}$ of complex numbers satisfying (4.1). Namely, given such a sequence $\boldsymbol{\nu}$, we

(1) construct the product $\tilde{F}$ of $(4.2)$;

(2) then calculate the residua $-c_{n}$ of $\tilde{F}$ at the points $\lambda_{n}$;

(3) construct sequences $\left(a_{n}\right)_{n \in I}$ and $\left(b_{n}\right)_{n \in I}$ via (4.7) and (4.8);

(4) determine the functions $\varphi$ and $\psi$ from their Fourier coefficients $a_{n}$ and $b_{n}$ via (2.4). 
As was noted at the beginning of this section, there are infinitely many such operators; all of them are fixed by the condition $\bar{a}_{n} b_{n}=c_{n}$ on the Fourier coefficient $a_{n}$ and $b_{n}$ of the functions $\varphi$ and $\psi$.

\section{Examples and Discussion}

We give here two examples illustrating that the results of the paper are in a sense optimal. For simplicity, we take the unperturbed operator $A$ to be defined in the Hilbert space $L_{2}(0,2 \pi)$ via

$$
A=\frac{1}{i} \frac{d}{d x}
$$

subject to the periodic boundary condition $y(0)=y(2 \pi)$. The spectrum of $A$ coincides with the set $\mathbb{Z}$, and a normalised eigenfunction $v_{n}$ corresponding to the eigenvalue $\lambda_{n}:=n$ is equal to $e^{i n x} / \sqrt{2 \pi}$. Therefore, the characteristic function of a generic rank-one perturbation $B$ of (2.2) has the form

$$
F(z)=\sum_{n \in \mathbb{Z}} \frac{c_{n}}{n-z}+1,
$$

where $c_{n}:=\bar{a}_{n} b_{n}$ is determined via the Fourier coefficients $a_{n}$ and $b_{n}$ of the functions $\varphi$ and $\psi$.

Example 5.1. Our first example shows that convergence of the series (3.1) is not guaranteed if the functions $\varphi$ and $\psi$ do not belong to $L_{2}(0,2 \pi)$. Namely, we take $a_{n}=a_{-n}=b_{n}=-b_{-n}=n^{-1 / 2}$ for $n \in \mathbb{N}$ and $a_{0}=b_{0}=0$; thus $c_{n}=n^{-1}$ for $n \neq 0$. To study the asymptotics of the corresponding eigenvalues $\mu_{n}$ of the operator $B$, we recall the equality [1, Ch. 5.2]

$$
\sum_{\substack{n \in \mathbb{Z} \\ n \neq 0}} \frac{1}{n(n-z)}=\frac{1}{z^{2}}-\frac{\pi}{z} \cot \pi z
$$

thus

$$
F(z)=\sum_{\substack{n \in \mathbb{Z} \\ n \neq 0}} \frac{1}{n(n-z)}+1=\frac{z^{2}+1}{z^{2}}-\frac{\pi}{z} \cot \pi z .
$$

It follows that $\mu_{n}$ are zeros of the equation

$$
\tan \pi z=\frac{\pi z}{z^{2}+1}
$$

and thus $\mu_{n}=\lambda_{n}+\varepsilon_{n}$ with $\varepsilon_{n} \rightarrow 0$ as $|n| \rightarrow \infty$; the relation

$$
\frac{\mu_{n}}{\varepsilon_{n}\left(\mu_{n}^{2}+1\right)}=\frac{\tan \pi \varepsilon_{n}}{\pi \varepsilon_{n}} \rightarrow 1
$$

as $|n| \rightarrow \infty$ now implies that $\varepsilon_{n} \mu_{n} \rightarrow 1$, and thus $\varepsilon_{n}=n^{-1}(1+o(1))$ as $|n| \rightarrow \infty$. As a result, the series (3.1) diverges. 
Example 5.2. Consider the rank-one perturbation $B$ of $A$ as in (2.2) with $\varphi$ and $\psi$ given by their Fourier coefficients $a_{0}=b_{0}=0$ and $a_{n}=a_{-n}=n^{-\beta / 2}$ and $b_{n}=b_{-n}=n^{-\beta / 2}$ for $n \in \mathbb{N}$, with $\beta>1$. We observe that the functions $\varphi$ and $\psi$ can be found explicitly via the fractional derivatives, cf. [39]. The corresponding characteristic function $F$ is equal to

$$
F(z)=1+\sum_{n \neq 0} \frac{|n|^{-\beta}}{n-z},
$$

and can be also represented as a product

$$
F(z)=\prod_{\substack{n \in \mathbb{Z} \\ n \neq 0}} \frac{\mu_{n}-z}{n-z}
$$

The proof of Theorem 3.1 (see (3.9)) shows that $\mu_{n}-n=O\left(|n|^{-\beta}\right)$ as $|n| \rightarrow \infty$. The residue of $F$ at the point $z=n$ is equal to $-|n|^{-\beta}$; on the other hand, it can be calculated as (cf. (4.4))

$$
\underset{z=n}{\operatorname{res}} F(z)=\left(n-\mu_{n}\right) \prod_{\substack{m \in \mathbb{Z} \\ m \neq n}} \frac{\mu_{m}-n}{m-n} .
$$

The infinite products in the above formula have been shown in the proof of Lemma 4.4 to be uniformly bounded in $n$ (cf. the reasoning following formula (4.6)). Therefore, we conclude that

$$
|n|^{-\beta} \leq C\left|\mu_{n}-n\right|,
$$

for a constant $C$ independent of $n$, so that

$$
\left|\mu_{n}-n\right| \asymp|n|^{-\beta} \text {. }
$$

Remark 5.3. The same arguments lead to conclusion that, for a generic rankone perturbation, $\left|\mu_{n}-\lambda_{n}\right| \asymp\left|a_{n} b_{n}\right|$ as $|n| \rightarrow \infty$. This allows us to control the decay of the offsets $\left|\mu_{n}-\lambda_{n}\right|$ through the products $\left|a_{n} b_{n}\right|$ of the Fourier coefficients of $\varphi$ and $\psi$ and vice versa.

Fix an arbitrary function $\varphi \in H$ and let $a_{n}$ be its Fourier coefficients in the orthonormal basis $\left(v_{n}\right)_{n \in I}$ of the eigenfunctions $v_{n}$ of $A$. Denote by $\ell_{1}(\varphi)$ the subspace of $\ell_{1}(I)$ consisting of all sequences $\mathbf{c}=\left(c_{n}\right)_{n \in I}$ of the form $c_{n}=x_{n} a_{n}$ with $\left(x_{n}\right)_{n \in I} \in \ell_{2}(I)$. The above analysis leads to the following uniqueness result:

Corollary 5.4. Given $\varphi \in H$, for every sequence $\varepsilon=\left(\varepsilon_{n}\right)_{n \in I} \in \ell_{1}(\varphi)$ there exists a function $\psi \in H$ such that the rank-one perturbation $B$ of the operator $A$ given by (2.2) has eigenvalues $\mu_{n}:=\lambda_{n}+\varepsilon_{n}, n \in I$, counting with multiplicities.

Such $\psi$ is unique if and only if none of $a_{n}$ vanishes; each $n \in I$ such that $a_{n}=0$ leaves the corresponding Fourier coefficient $b_{n}$ undetermined and thus increases by one the degree of freedom of the set of all such $\psi$.

The roles of $\varphi$ and $\psi$ can be interchanged. 
We conclude the paper with some comments on the results obtained. Most of the analysis of [15] and of this paper can be generalised in a straightforward way to the case of a normal operator $A$. The most crucial properties and facts used are

(a) the spectrum of $A$ is simple and separated;

(b) the eigenvectors form an orthonormal basis (or even a Riesz basis) of $H$;

(c) the spectral theorem allowing to represent the characteristic function $F$ of a rank-one perturbation $B$ in the form (2.5).

Some care should be given to properly choose the regions $Q_{k}$ in Sects. 3 and 4 , but otherwise the arguments remain valid and establish Theorems 3.1 and 4.1 , i.e., justify the possibility to enumerate the spectrum of $B$ so that series (3.1) converges and, for every sequence $\left(\nu_{n}\right)_{n \in I}$ satisfying (4.1), to construct a rank-one perturbation $B$ of $A$ whose spectrum is given by that sequence counting with multiplicities.

In the special case of a self-adjoint rank-one perturbation (2.2) with $\psi=\alpha \varphi$ and $\alpha \in \mathbb{R}$, the resulting spectrum of $B$ is simple outside $\sigma_{0}(A)$, of geometric multiplicity at most 2 at the points of $\sigma_{0}(A)$, and the eigenvalues $\sigma_{1}(A)$ and $\sigma_{1}(B)$ strictly interlace, i.e., between every two consecutive eigenvalues from $\sigma_{1}(A)$ there is a unique eigenvalue from $\sigma_{1}(B)$ and, vice versa, between every two consecutive eigenvalues from $\sigma_{1}(B)$ there is a unique eigenvalue from $\sigma_{1}(A)$. This interlacing property follows from the minmax principle [34]; moreover, for $\alpha>0$ we have $\lambda_{n}<\mu_{n}$ for all $n \in I_{1}$; the signs are reversed if $\alpha<0$. Given $\left(\mu_{n}\right)_{n \in I}$ satisfying the interlacing property, convergence of the series (3.1) is a necessary and sufficient condition on the spectrum of a self-adjoint rank-one perturbation $B$ of $A$.

Open Access. This article is licensed under a Creative Commons Attribution 4.0 International License, which permits use, sharing, adaptation, distribution and reproduction in any medium or format, as long as you give appropriate credit to the original author(s) and the source, provide a link to the Creative Commons licence, and indicate if changes were made. The images or other third party material in this article are included in the article's Creative Commons licence, unless indicated otherwise in a credit line to the material. If material is not included in the article's Creative Commons licence and your intended use is not permitted by statutory regulation or exceeds the permitted use, you will need to obtain permission directly from the copyright holder. To view a copy of this licence, visit http:// creativecommons.org/licenses/by/4.0/.

Publisher's Note Springer Nature remains neutral with regard to jurisdictional claims in published maps and institutional affiliations.

\section{References}

[1] Ahlfors, L.V.: Complex Analysis. An Introduction to the Theory of Analytic Functions of One Complex Variable, 3rd edn. McGraw-Hill Book Co., New York (1978) 
[2] Albeverio, S., Gesztesy, F., Høegh-Krohn, R., Holden, H.: Solvable Models in Quantum Mechanics. With an Appendix by Pavel Exner, 2nd edn. AMS Chelsea Publishing, Providence, RI (2005)

[3] Albeverio, S., Konstantinov, A., Koshmanenko, V.: Decompositions of singular continuous spectra of $\mathscr{H}_{-2}$-class rank one perturbations. Integral Equ. Oper. Theory 52(4), 455-464 (2005)

[4] Albeverio, S., Koshmanenko, V., Kurasov, P., Nizhnik, L.: On approximations of rank one $\mathscr{H}_{-2}$-perturbations. Proc. Am. Math. Soc. 131(5), 1443-1452 (2003)

[5] Albeverio, S., Koshmanenko, V.: Singular rank one perturbations of self-adjoint operators and Krein theory of self-adjoint extensions. Potential Anal. 11, 279287 (1999)

[6] Albeverio, S., Kurasov, P.: Rank one perturbations, approximations and selfadjoint extensions. J. Funct. Anal. 148, 152-169 (1997)

[7] Albeverio, S., Kurasov, P.: Rank one perturbations of not semibounded operators. Integral Equ. Oper. Theory 27, 379-400 (1997)

[8] Albeverio, S., Kurasov, P.: Finite rank perturbations and distribution theory. Proc. Am. Math. Soc. 127, 1151-1161 (1999)

[9] Albeverio, S., Kurasov, P.: Singular Perturbations of Differential Operators: Schrödinger-Type Operators. Cambridge University Press, Cambridge (2000)

[10] Albeverio, S., Kuzhel, S., Nizhnik, L.: On the perturbation theory of self-adjoint operators. Tokyo J. Math. 31(2), 273-292 (2008)

[11] Baragaña, I., Roca, A.: Fixed rank perturbations of regular matrix pencils. Linear Algebra Appl. 589, 201-221 (2020)

[12] Behrndt, J., Leben, L., Peria, F.M., Möws, R., Trunk, C.: The effect of finite rank perturbations on Jordan chains of linear operators. Linear Algebra Appl. 479, 118-130 (2015)

[13] Behrndt, J., Leben, L., Peria, F.M., Möws, R., Trunk, C.: Sharp eigenvalue estimates for rank one perturbations of nonnegative operators in Krein spaces. J. Math. Anal. Appl. 439(2), 864-895 (2016)

[14] Behrndt, J., Möws, R., Trunk, C.: On finite rank perturbations of selfadjoint operators in Krein spaces and eigenvalues in spectral gaps. Complex Anal. Oper. Theory 8(4), 925-936 (2014)

[15] Dobosevych, O., Hryniv, R.: Spectra of rank-one perturbations of self-adjoint operators. Linear Algebra Appl. 609, 339-364 (2021)

[16] Dudkin, M., Vdovenko, T.: On nonsymmetric rank one singular perturbations of selfadjoint operators. Methods Funct. Anal. Topol. 22(2), 137-151 (2016)

[17] Farrell, P.E.: The number of distinct eigenvalues of a matrix after perturbation. SIAM J. Matrix Anal. Appl. 37(2), 572-576 (2016)

[18] Golovaty, Y.: Schrödinger operators with singular rank-two perturbations and point interactions. Integral Equ. Oper. Theory 90(5), 1-24 (2018)

[19] Gernandt, H., Trunk, C.: Eigenvalue placement for regular matrix pencils with rank one perturbations. SIAM J. Matrix Anal. Appl. 38(1), 134-154 (2017)

[20] Homa, M., Hryniv, R.: Spectra of $\mathcal{P} \mathcal{T}$-symmetric operators under rank-one perturbations. J. Phys. A Math. Theor. 53, 375202 (2020)

[21] Hörmander, L., Melin, A.: A remark on perturbations of compact operators. Math. Scand. 75, 255-262 (1994) 
[22] Kato, T.: Perturbation Theory for Linear Operators. Springer-Verlag, Berlin (1995) (Reprint of the 1980 edition. Classics in Mathematics)

[23] Krupnik, M.: Changing the spectrum of an operator by perturbation. Linear Algebra Appl. 167, 113-118 (1992)

[24] Kurasov, P: Singular and supersingular perturbations: Hilbert space methods. In: Spectral Theory of Schrödinger Operators, 185-216, Contemp. Math., 340. Amer. Math. Soc., Providence, RI (2004)

[25] Kurasov, P., Luger, A., Neuner, Ch.: On supersingular perturbations of nonsemibounded self-adjoint operators. J. Oper. Theory 81(1), 195-223 (2019)

[26] Kuzhel, S., Nizhnik, L.: Finite rank self-adjoint perturbations. Methods Funct. Anal. Topol. 12(3), 243-253 (2006)

[27] Mehl, C., Mehrmann, V., Ran, A.C.M., Rodman, L.: Eigenvalue perturbation theory of classes of structured matrices under generic structured rank one perturbations. Linear Algebra Appl. 435, 687-716 (2011)

[28] Mehl, C., Mehrmann, V., Ran, A.C.M., Rodman, L.: Perturbation theory of selfadjoint matrices and sign characteristics under generic structured rank one perturbations. Linear Algebra Appl. 436, 4027-4042 (2012)

[29] Mehl, C., Mehrmann, V., Ran, A.C.M., Rodman, L.: Jordan forms of real and complex matrices under rank one perturbations. Oper. Matrices 7(2), 381-398 (2013)

[30] Mehl, C., Mehrmann, V., Ran, A.C.M., Rodman, L.: Eigenvalue perturbation theory under generic rank one perturbations: symplectic, orthogonal, and unitary matrices. BIT 54, 219-255 (2014)

[31] Mehl, C., Mehrmann, V., Ran, A.C.M., Rodman, L.: Eigenvalue perturbation theory of structured real matrices and their sign characteristics under generic structured rank-one perturbations. Linear Multilinear Algebra 64(3), 527-556 (2016)

[32] Mehl, C., Mehrmann, V., Wojtylak, M.: Parameter-dependent rank-one perturbations of singular Hermitian or symmetric pencils. SIAM J. Matrix Anal. Appl. 38(1), 72-95 (2017)

[33] Moro, J., Dopico, F.: Low rank perturbation of Jordan structure. SIAM J. Matrix Anal. Appl. 25, 495-506 (2003)

[34] Reed, M., Simon, B.: Methods of Modern Mathematical Physics IV: Analysis of Operators. Academic Press, London (1978)

[35] Savchenko, S.V.: Typical changes in spectral properties under perturbations by a rank-one operator, Mat. Zametki 74, 590-602 (in Russian). Engl. translat. in Math. Notes 74(2003), 557-568 (2003)

[36] Savchenko, S.V.: On the change in the spectral properties of a matrix under a perturbation of a sufficiently low rank, Funktsional. Anal. Prilozhen. 38: 85-88 (in Russian). Engl. translat. in Funct. Anal. Appl. 38(2004), 69-71 (2004)

[37] Simon, B.: Spectral analysis of rank one perturbations and applications. CRM Proc. Lecture Notes 8, 109-149 (1995)

[38] Sosa, F., Moro, J., Mehl, C.: First order structure-preserving perturbation theory for eigenvalues of symplectic matrices. SIAM J. Matrix Anal. Appl. 41(2), 657-690 (2020)

[39] Tseng, C.-C., Pei, S.-C., Hsia, S.-C.: Computation of fractional derivatives using Fourier transform and digital FIR differentiator. Signal Process. 80(1), 151-159 (2000) 
Oles Dobosevych and Rostyslav Hryniv ( $ه)$

Ukrainian Catholic University

2a Kozelnytska str

79026 Lviv

Ukraine

e-mail: rhryniv@ucu.edu .ua

Oles Dobosevych

e-mail: dobosevych@ucu.edu.ua

Rostyslav Hryniv

University of Rzeszów

1 Pigonia str.

35-959 Rzeszow

Poland

e-mail: rhryniv@ur.edu.pl

Received: July 12, 2020.

Revised: March 21, 2021. 